\title{
Covering Spheres with Spheres
}

\section{Ilya Dumer}

Received: 1 June 2006 / Revised: 14 July 2007 / Published online: 2

September 2007

(C) Springer Science+Business Media, LLC 2007

\begin{abstract}
Given a sphere of any radius $r$ in an $n$-dimensional Euclidean space, we study the coverings of this sphere with solid spheres of radius one. Our goal is to design a covering of the lowest covering density, which defines the average number of solid spheres covering a point in a bigger sphere. For growing dimension $n$, we design a covering that gives the covering density of order $(n \ln n) / 2$ for a sphere of any radius $r>1$ and a complete Euclidean space. This new upper bound reduces two times the order $n \ln n$ established in the classic Rogers bound.
\end{abstract}

\section{Introduction}

Spherical coverings. Let $B_{r}^{n}(\mathbf{x})$ be a ball (solid sphere) of radius $r$ centered at some point $\mathbf{x}=\left(x_{1}, \ldots, x_{n}\right)$ of an $n$-dimensional Euclidean space $\mathbb{R}^{n}$ :

$$
B_{r}^{n}(\mathbf{x}) \stackrel{\text { def }}{=}\left\{\mathbf{z} \in \mathbb{R}^{n} \mid \sum_{i=1}^{n}\left(z_{i}-x_{i}\right)^{2} \leq r^{2}\right\} .
$$

We also use a simpler notation $B_{r}^{n}$ if a ball is centered at the origin $\mathbf{x}=0$. For any subset $A \subseteq \mathbb{R}^{n}$, we say that a subset $\operatorname{Cov}(A, \varepsilon) \subseteq \mathbb{R}^{n}$ forms an $\varepsilon$-covering (an $\varepsilon$-net) of $A$ if $A$ is contained in the union of the balls of radius $\varepsilon$ centered at points $\mathbf{x} \in \operatorname{Cov}(A, \varepsilon)$. In this case, we use notation

$$
\operatorname{Cov}(A, \varepsilon): A \subseteq \bigcup_{\mathbf{x} \in \operatorname{Cov}(A, \varepsilon)} B_{\varepsilon}^{n}(\mathbf{x}) .
$$

By changing the scale in $\mathbb{R}^{n}$, we can always consider the rescaled set $A / \varepsilon$ and the new covering $\operatorname{Cov}(A / \varepsilon, 1)$ with unit balls $B_{1}^{n}(\mathbf{x})$. Without loss of generality, below

\footnotetext{
I. Dumer ( $\square)$

University of California, Riverside, USA

e-mail: dumer@ee.ucr.edu
} 
we consider these (unit) coverings. One of the classical problems is to obtain tight bounds on the covering size $\left|\operatorname{Cov}\left(B_{r}^{n}, 1\right)\right|$ for any ball $B_{r}^{n}$ of radius $r$ and dimension $n$.

Another related covering problem arises for a sphere

$$
S_{r}^{n} \stackrel{\text { def }}{=}\left\{\mathbf{z} \in \mathbb{R}^{n+1} \mid \sum_{i=1}^{n+1} z_{i}^{2}=r^{2}\right\} .
$$

Then a unit ball $B_{1}^{n+1}(\mathbf{x})$ intersects this sphere with a spherical cap

$$
C_{r}^{n}(\rho, \mathbf{y})=S_{r}^{n} \cap B_{1}^{n+1}(\mathbf{x}),
$$

which has some center $\mathbf{y} \in S_{r}^{n}$, half-chord $\rho \leq 1$, and the corresponding half-angle $\alpha=\arcsin \rho / r$. The biggest possible cap $C_{r}^{n}(1, \mathbf{y})$ is obtained if the center $\mathbf{x}$ of the corresponding ball $B_{1}^{n+1}(\mathbf{x})$ is centered at the distance

$$
\|\mathbf{x}\|=\sqrt{r^{2}-1}
$$

from the origin. To obtain a minimal covering, we shall consider the biggest caps $C_{r}^{n}(1, \mathbf{y})$ assuming that all the centers $\mathbf{x}$ satisfy (1).

Covering density. Given a set $A \subseteq \mathbb{R}^{n}$, let $|A|$ denote $n$-dimensional volume (Lebesque measure) of $A$. We then consider any unit $\operatorname{covering} \operatorname{Cov}(A, 1)$ and define minimum covering density

$$
\vartheta(A)=\min _{\operatorname{Cov}(A, 1)} \sum_{\mathbf{x} \in \operatorname{Cov}(A, 1)} \frac{\left|B_{1}^{n}(\mathbf{x}) \cap A\right|}{|A|} .
$$

Minimal coverings have been long studied for the spheres $S_{r}^{n}$ and the balls $B_{r}^{n}$. The celebrated Coxeter-Few-Rogers lower bound [1] shows that for a sufficiently large ball $B_{r}^{n}$,

$$
\vartheta\left(S_{r}^{n}\right) \geq c_{0} n
$$

Here and below $c_{i}$ denote some universal constants. A similar result also holds for any sphere $S_{r}^{n}$ of radius $r \geq n$ (see Example 6.3 in [4]).

Various upper bounds on the minimum covering density are obtained for $B_{r}^{n}$ and $S_{r}^{n}$ by Rogers in the classic papers [2] and [3]. In particular, it follows from these papers that for a sufficiently large radius $r$, a ball $B_{r}^{n}$ and a sphere $S_{r}^{n}$ can be covered with density

$$
\vartheta \leq\left(1+\frac{\ln \ln n}{\ln n}+\frac{5}{\ln n}\right) n \ln n .
$$

Despite recent improvements obtained in [4] and [5], respectively, for spheres $S_{r}^{n}$ and balls $B_{r}^{n}$ of a relatively small radius $r$, the Rogers bound (3) is still the best asymptotic bound known for sufficiently large spheres, balls, and complete spaces $\mathbb{R}^{n}$ of growing dimension $n$. 
For a sphere $S_{r}^{n}$ of any dimension $n \geq 3$ and an arbitrary radius $r>1$, the best universal upper bound known to date is obtained in [4] (see Corollary 1.2 and Remark 5.1):

$$
\vartheta\left(S_{r}^{n}\right) \leq\left(1+\frac{2}{\ln n}\right)\left(1+\frac{\ln \ln n}{\ln n}+\frac{\sqrt{e}}{n \ln n}\right) n \ln n .
$$

Our main result is presented in Theorem 1, which reduces about two times the present upper bounds (3) and (4) for $n \rightarrow \infty$.

Theorem 1 Unit balls can cover a sphere $S_{r}^{n}$ of any radius $r>1$ and any dimension $n \geq 3$ with density

$$
\vartheta\left(S_{r}^{n}\right) \leq\left(\frac{1}{2}+\frac{2 \ln \ln n}{\ln n}+\frac{5}{\ln n}\right) n \ln n .
$$

For $n \rightarrow \infty$, there exists $o(1) \rightarrow 0$ such that

$$
\vartheta\left(S_{r}^{n}\right) \leq\left(\frac{1}{2}+\left(\frac{3}{2}+o(1)\right) \frac{\ln \ln n}{\ln n}\right) n \ln n .
$$

The following corollary to Theorem 1 (see also [8]) shows that the Rogers bound can also be reduced about two times for the coverings of complete Euclidean spaces $\mathbb{R}^{n}$.

Corollary 2 For $n \rightarrow \infty$, unit balls can cover the entire Euclidean space $\mathbb{R}^{n}$ with density

$$
\vartheta\left(\mathbb{R}^{n}\right) \leq\left(\frac{1}{2}+o(1)\right) n \ln n
$$

\section{Preliminaries: Embedded Coverings}

In this section, we obtain the estimates on $\vartheta\left(S_{r}^{n}\right)$ that are similar to (3) and (4). However, we will introduce here a slightly different technique of embedded coverings that will be substantially extended in Section 3 to improve the former bounds (3) and (4). We will also employ most of our calculations performed in this section.

Consider a sphere $S_{r}^{n}$ of some dimension $n \geq 3$ and radius $r>1$. We use notation $C(\rho, \mathbf{y})$ for a cap $C_{r}^{n}(\rho, \mathbf{y})$ whenever parameters $n$ and $r$ are fixed; we also use a shorter notation $C(\rho)$ when a specific center $\mathbf{y}$ is of no importance. In this case, $\operatorname{Cov}(\rho)$ will denote any covering of $S_{r}^{n}$ with spherical caps $C(\rho)$. By definition, a covering $\operatorname{Cov}(\rho)$ has covering density

$$
\vartheta_{\rho}=\Omega_{\rho}|\operatorname{Cov}(\rho)|
$$

where $\Omega_{\rho}$ is the fraction of the surface of the sphere $S_{r}^{n}$ covered by a cap $C(\rho)$,

$$
\Omega_{\rho}=\frac{|C(\rho)|}{\left|S_{r}^{n}\right|}
$$


For any $\tau<\rho \leq 1$, we extensively use inequality (see Corollary 3.2 (ii) in [4]):

$$
\Omega_{\tau} \geq \Omega_{\rho}\left(\frac{\tau}{\rho}\right)^{n}
$$

and its particular version $\Omega_{\tau} \geq \Omega_{1} \tau^{n}$ obtained for $\rho=1$. We begin with two preliminary lemmas, which will simplify our calculations. Let $f_{1}(x)$ and $f_{2}(x)$ be two positive differentiable functions. We say that $f_{1}(x)$ moderates $f_{2}(x)$ for $x \geq a$ if for all $x \geq a$,

$$
\frac{f_{1}^{\prime}(x)}{f_{1}(x)} \geq \frac{f_{2}^{\prime}(x)}{f_{2}(x)}
$$

Lemma 3 Consider $m$ functions $f_{i}(x)$ such that $f_{1}(x)$ moderates each function $f_{i}(x), i \geq 2$, for $x \geq a$. Then inequality

$$
f_{1}(x) \geq \sum_{i=2}^{m} f_{i}(x)
$$

holds for any $x \geq a$ if it is valid for $x=a$.

Proof Note that $f_{i}(x)=f_{i}(a) \exp \left\{s_{i}(x)\right\}$, where

$$
s_{i}(x) \stackrel{\text { def }}{=} \int_{a}^{x} \frac{f_{i}^{\prime}(t)}{f_{i}(t)} d t
$$

Also, $s_{i}(x) \leq s_{1}(x)$ for all $i \geq 2$. Therefore,

$$
f_{1}(x) \geq \sum_{i=2}^{m} f_{i}(a) \exp \left\{s_{1}(x)\right\} \geq \sum_{i=2}^{m} f_{i}(a) \exp \left\{s_{i}(x)\right\}=\sum_{i=2}^{m} f_{i}(x),
$$

which completes the proof.

Lemma 4 For any $n \geq 4$,

$$
\left(1-\frac{1}{n \ln n}\right)^{-n}<1+1 / \ln n+1 / \ln ^{2} n
$$

Proof For $n=4, \ldots, 7$, the above inequality is verified numerically. Let $t=\frac{1}{\ln n}$. We take the logarithm of the left part of (9) and use power series. This gives the following inequalities

$$
-n \ln \left(1-\frac{t}{n}\right)=\sum_{i=1}^{\infty} \frac{t^{i}}{n^{i-1} i}<t+t^{2} \sum_{i=1}^{\infty}\left(\frac{1}{2 n}\right)^{i} \leq t+\frac{t^{2}}{15}
$$

Here we observe that $t<\frac{1}{2}$ for $n \geq 8$, and replace the left-hand side of the first inequality with geometric series in its right-hand side, which in turn is bounded by 
taking $n=8$. We also note that for any $n \geq 8$,

$$
t+\frac{t^{2}}{15}<\frac{1}{2}
$$

For any $z<\frac{1}{2}$ we can also use inequality

$$
e^{z}=\sum_{i=1}^{\infty} \frac{z^{i}}{i !} \leq 1+z+\frac{2 z^{2}}{3}
$$

By combining (10) and (12), we obtain the required estimate

$$
\left(1-\frac{1}{n \ln n}\right)^{-n}<1+\left(t+\frac{t^{2}}{15}\right)+\frac{2}{3}\left(t+\frac{t^{2}}{15}\right)^{2}<1+t+t^{2}
$$

The latter inequality can be verified for any $t<1 / 2$ directly, or by applying Lemma 3 for $t=\frac{1}{z}$ and $z \geq 2$.

An embedded algorithm. We employ the following parameters

$$
\begin{gathered}
\varepsilon=\frac{1}{n \ln n}, \quad \rho=1-\varepsilon, \\
\lambda=1+\frac{\ln \ln n}{\ln n}+\frac{2}{n} .
\end{gathered}
$$

To design a covering $\operatorname{Cov}(1)$, we also use another covering

$$
\operatorname{Cov}(\varepsilon): S_{r}^{n} \subseteq \bigcup_{\mathbf{u} \in \operatorname{Cov}(\varepsilon)} C(\varepsilon, \mathbf{u})
$$

with smaller caps $C(\varepsilon, \mathbf{u})$. In our first step, we randomly choose $N$ points $\mathbf{y} \in S_{r}^{n}$, where

$$
\frac{\lambda n \ln n}{\Omega_{\rho}}-1<N \leq \frac{\lambda n \ln n}{\Omega_{\rho}} .
$$

Consider the set $\{C(\rho, \mathbf{y})\}$ of $N$ caps. In the second step, we take all centers $\mathbf{u}^{\prime} \in$ $\operatorname{Cov}(\varepsilon)$ that are left uncovered by the set $\{C(\rho, \mathbf{y})\}$ and form the extended set

$$
\{\mathbf{x}\}=\{\mathbf{y}\} \cup\left\{\mathbf{u}^{\prime}\right\} .
$$

This set $\{\mathbf{x}\}$ covers the entire set $\operatorname{Cov}(\varepsilon)$ and therefore forms a unit covering, if the caps $C(\rho, \mathbf{x})$ are expanded to the caps $C(1, \mathbf{x})$,

$$
\operatorname{Cov}(1): S_{r}^{n} \subseteq \bigcup_{\mathbf{x} \in\{\mathbf{x}\}} C(1, \mathbf{x})
$$

Lemma 5 For any $n \geq 8$, covering $\{\mathbf{x}\}$ has density

$$
\vartheta_{*} \leq\left(1+\frac{\ln \ln n}{\ln n}+\frac{3}{\ln n}\right) n \ln n .
$$


Proof Any point $\mathbf{u}$ is covered by some cap $C(\rho, \mathbf{y})$ with probability $\Omega_{\rho}$. Our goal is to estimate the expected number $N^{\prime}$ of centers $\mathbf{u}^{\prime}$ left uncovered after $N$ trials. Here

$$
N^{\prime}=\left(1-\Omega_{\rho}\right)^{N} \cdot|\operatorname{Cov}(\varepsilon)| .
$$

Note that $1-\Omega_{\rho} \leq e^{-\Omega_{\rho}\left(1+\Omega_{\rho} / 2\right)}$. Also, according to estimate (15), $N \Omega_{\rho}>\lambda n \ln n-$ $\Omega_{\rho}$. Thus,

$$
\left(1-\Omega_{\rho}\right)^{N} \leq e^{-\left(\lambda n \ln n-\Omega_{\rho}\right)\left(1+\Omega_{\rho} / 2\right)} \leq e^{-\lambda n \ln n} .
$$

Here the second inequality holds since $\Omega_{\rho}<\Omega_{1}<1 / 2$, whereas $\lambda n \ln n>16$.

Next, we estimate the covering size $|\operatorname{Cov}(\varepsilon)|$. Let this covering have some density $\vartheta_{\varepsilon}$. Then inequality (8) gives the estimates

$$
\begin{gathered}
|\operatorname{Cov}(\varepsilon)|=\vartheta_{\varepsilon} / \Omega_{\varepsilon} \leq(n \ln n)^{n} \vartheta_{\varepsilon} / \Omega_{1}, \\
N^{\prime} \leq e^{-\lambda n \ln n}(n \ln n)^{n} \vartheta_{\varepsilon} / \Omega_{1} \leq \vartheta_{\varepsilon} /\left(n^{2} \Omega_{1}\right) .
\end{gathered}
$$

According to our design, there exists a covering $\{\mathbf{x}\}$ with caps $C(1, \mathbf{x})$ that has size at most $N+N^{\prime}$. Next, we combine last inequality with (15), and estimate the covering density of $\{\mathbf{x}\}$ :

$$
\vartheta_{1}=\Omega_{1}\left(N+N^{\prime}\right) \leq(\lambda n \ln n) \Omega_{1} / \Omega_{\rho}+\vartheta_{\varepsilon} / n^{2} .
$$

Finally, we estimate $\Omega_{1} / \Omega_{\rho}$ using inequalities (8) and (9):

$$
\Omega_{1} / \Omega_{\rho} \leq(1-\varepsilon)^{-n} \leq 1+1 / \ln n+1 / \ln ^{2} n .
$$

Thus, we can rewrite our estimate $\vartheta_{1}$ as

$$
\vartheta_{1} \leq \vartheta_{*}\left(1-1 / n^{2}\right)+\vartheta_{\varepsilon} / n^{2}
$$

using notation

$$
\vartheta_{*}=\lambda n \ln n \frac{1+1 / \ln n+1 / \ln ^{2} n}{1-1 / n^{2}} .
$$

For any given $n$, bound (20) only depends on the density $\vartheta_{\varepsilon}$. Now let us assume that $\vartheta_{1}$ meets some known upper bound $\tilde{\vartheta}_{1}$ valid for all (or sufficiently large) radii $r$. For example, we can use (4) or any weaker estimate. Next, we can change the scale in $\mathbb{R}^{n+1}$ and replace a covering $\operatorname{Cov}(1)$ of a sphere $S_{r / \varepsilon}^{n}$ with the $\operatorname{covering} \operatorname{Cov}(\varepsilon)$ of the sphere $S_{r}^{n}$. This rescaling shows that we can replace $\vartheta_{\varepsilon}$ in (20) with any known upper bound $\tilde{\vartheta}_{1}$. In turn, inequality (20) shows that the bound $\tilde{\vartheta}_{1}$ will be further reduced by this replacement as long as $\tilde{\vartheta}_{1}>\vartheta_{*}$. Thus, this iteration process yields the upper bound $\vartheta_{1} \leq \vartheta_{*}$.

Our final step is to verify that the upper bound $\vartheta_{*}$ of (21) also satisfies inequality (16). Here we use Lemma 3. Then straightforward calculations show that the right-hand side of (16) exceeds the right-hand side of (21) for $n=8$ and moderates it for $n \geq 8$, due to the bigger remaining term $3 / \ln n$ in (16). 
Remark More detailed arguments show that (16) holds for $n \geq 3$, whereas for $n \geq 8$,

$$
\vartheta_{*} \leq\left(1+\frac{\ln \ln n}{\ln n}+\frac{2}{\ln n}\right) n \ln n .
$$

\section{New Covering Algorithm for a Sphere $S_{r}^{n}$}

Covering design. In this section, we obtain a covering of the sphere $S_{r}^{n}$ with asymptotic density $(n \ln n) / 2$. The new design will use both the former covering $\operatorname{Cov}(\varepsilon)$ (with slightly different parameters) and another covering $\operatorname{Cov}(\mu)$ with a larger radius $\mu$ that has asymptotic order of $n^{-1 / 2}$. Namely, we use parameters

$$
\begin{aligned}
& \varepsilon=\frac{1}{2 n \ln n}, \\
& \rho=1-\varepsilon, \\
& \beta=\frac{1}{2}+2 \frac{\ln \ln n}{\ln n}, \\
& \lambda=\beta+\frac{5}{2} \frac{1}{\ln n}, \\
& \mu=\frac{n^{-\beta}}{2 \sqrt{3}}, \\
& d=1-2 \varepsilon-\mu^{2}
\end{aligned}
$$

and proceed as follows.

A. Let a sphere $S_{r}^{n}$ be covered with two different coverings $\operatorname{Cov}(\mu)$ and $\operatorname{Cov}(\varepsilon)$ :

$$
\begin{aligned}
& \operatorname{Cov}(\mu): S_{r}^{n} \subseteq \bigcup_{\mathbf{z} \in \operatorname{Cov}(\mu)} C(\mu, \mathbf{z}), \\
& \operatorname{Cov}(\varepsilon): S_{r}^{n} \subseteq \bigcup_{\mathbf{u} \in \operatorname{Cov}(\varepsilon)} C(\varepsilon, \mathbf{u}) .
\end{aligned}
$$

We assume that both coverings have the former density $\vartheta_{*}$ of (16) or less.

B. Randomly choose $N$ points $\mathbf{y} \in S_{r}^{n}$ and consider the corresponding spherical caps $C(\rho, \mathbf{y})$, where

$$
\frac{\lambda n \ln n}{\Omega_{d}}-1<N \leq \frac{\lambda n \ln n}{\Omega_{d}} .
$$

C. Let $C(\mu, \overline{\mathbf{z}})$ be any cap in $\operatorname{Cov}(\mu)$ that contains at least one center $\mathbf{u} \in \operatorname{Cov}(\varepsilon)$ not covered by the $\rho$-caps. We consider all such centers $\overline{\mathbf{z}}$ and form the joint set $\{\mathbf{x}\}=\{\mathbf{y}\} \cup\{\overline{\mathbf{z}}\}$. This set covers $\operatorname{Cov}(\varepsilon)$ with $\rho$-caps and therefore forms the required covering

$$
\operatorname{Cov}(1): S_{r}^{n} \subseteq \bigcup_{\mathbf{x} \in\{\mathbf{x}\}} C(1, \mathbf{x}) .
$$

We now proceed with preliminary discussion, which outlines the main steps of the proof. 
Outline of the proof. Let us first assume that we keep the design of Section 2 but apply it to the new covering $\operatorname{Cov}(\mu)$ instead of $\operatorname{Cov}(\varepsilon)$. This will require taking $\rho=1-\mu$ to cover the centers of the caps $C(\mu, \mathbf{z})$ and then expanding $\rho$ to 1 to cover the whole $\mu$-caps. Contrary to our former choice of $\rho=1-\varepsilon$ in (19), this expansion will exponentially increase the covering density. Namely, straightforward calculations show that

$$
\Omega_{1} / \Omega_{1-\varepsilon} \rightarrow 1, \quad \Omega_{1} / \Omega_{1-\mu}=\exp \left\{n^{1 / 2}\right\}, \quad n \rightarrow \infty .
$$

To circumvent this problem, we keep $\rho=1-\varepsilon$ in (22) but change our design as follows.

1. Given any cap $C(\mu, \mathbf{z})$, we say that a cap $C(\rho, \mathbf{y})$ is $d$-close if $\mathbf{y}$ falls within distance $d<\rho$ to $\mathbf{z}$. In our proof, we refine the selection of the caps $C(\rho, \mathbf{y})$ and count only $d$-close caps, instead of the $\rho$-close caps considered in Section 2. It is easy to verify that distance $d$ of (22) is so close to $\rho$ that

$$
\Omega_{\rho} / \Omega_{d} \rightarrow 1, \quad n \rightarrow \infty
$$

For this reason, counting only $d$-close caps instead of the former $\rho$-close caps will carry no overhead to the covering size (23).

2. On the other hand, we will show in Lemma 6 that the $\mu$-cap becomes almost completely covered by a cap $C(\rho, \mathbf{y})$ when the latter becomes $d$-close instead of $\rho$-close. Namely, only a vanishing fraction $\omega \approx \exp \left(-\frac{3}{2} \ln ^{2} n\right)$ of a $\mu$-cap is left uncovered in this case.

3. We shall also use the fact that a typical $\mu$-cap is covered by multiple $d$-close caps. According to (23), the average number $\Omega_{d} N$ of these caps has the exact order of $\lambda n \ln n$ :

$$
\lambda n \ln n-\Omega_{d}<\Omega_{d} N \leq \lambda n \ln n .
$$

In our proof, we first define insufficiently covered $\mu$-caps. Namely, we call a cap $C\left(\mu, \mathbf{z}^{\prime}\right)$ non-saturated if it has only $s$ or fewer $d$-close caps, where

$$
s=\lfloor n / q\rfloor, \quad q=3 \ln \ln n .
$$

This choice of $s$ will achieve two goals.

4. We prove in Lemma 7 that non-saturated caps typically form a very small fraction of order $\exp [-\lambda n \ln n]$ among all $\mu$-caps. On the other hand, it is easy to see that the quantity

$$
|\operatorname{Cov}(\mu)| \leq \vartheta_{*} / \Omega_{\mu}
$$

exceeds $N$ by the exponential factor $\Omega_{d} / \Omega_{\mu} \sim \exp [\beta n \ln n]$ or less. Then our choice of $\lambda$ and $\beta$ in (22) gives the expected number $N^{\prime}=o(N)$ of non-saturated caps. Thus, non-saturated caps typically form a vanishing fraction of not only $\mu$-caps but also $\rho$-caps.

5. Next, we proceed with saturated $\mu$-caps and count all those centers $\mathbf{u}^{\prime \prime} \in \operatorname{Cov}(\varepsilon)$ that are left uncovered by random $\rho$-caps. All caps $C\left(\mu, \mathbf{z}^{\prime \prime}\right)$ that contain uncovered centers $\mathbf{u}^{\prime \prime}$ are called porous. For a given $s$, we show in Lemma 8 that 
Fig. 1 Two intersecting caps $C(\mu, \mathbf{Z})$ and $C(\rho, \mathbf{Y})$ with bases PQRSA and PMRTB

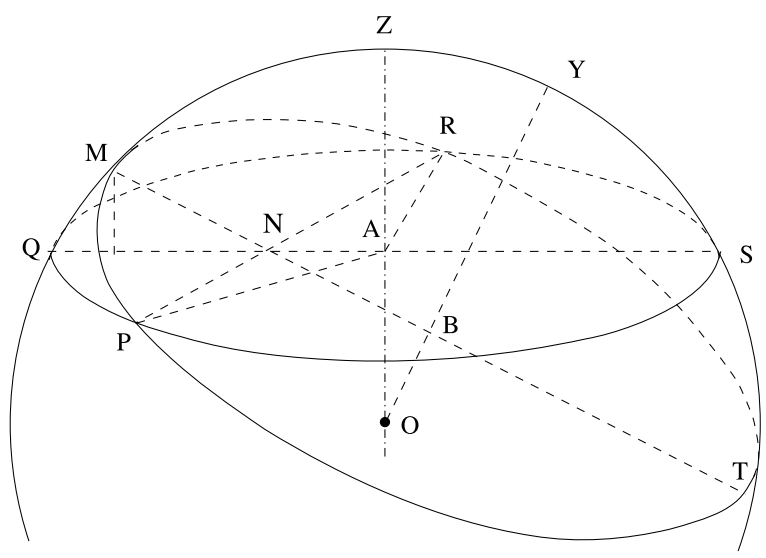

the set $\left\{\mathbf{u}^{\prime \prime}\right\}$ forms a very small portion of $\operatorname{Cov}(\varepsilon)$, with expected fraction of $\omega^{s+1} \sim \exp \left[-\frac{3}{2} n \ln n\right]$. Note that the quantity

$$
|\operatorname{Cov}(\varepsilon)| \leq \vartheta_{*} / \Omega_{\varepsilon}
$$

exceeds $N$ by the exponential factor $\Omega_{d} / \Omega_{\varepsilon} \sim \exp [n \ln n]$ or less. Therefore, the expected size of $\left\{\mathbf{u}^{\prime \prime}\right\}$ is $N^{\prime \prime}=o(N)$.

6. Finally, the centers of all non-saturated and porous caps are combined into the set $\overline{\mathbf{z}}=\left\{\mathbf{z}^{\prime}, \mathbf{z}^{\prime \prime}\right\}$. Then the set $\{\mathbf{x}\}=\{\mathbf{y}, \overline{\mathbf{z}}\}$ completely covers the set $\operatorname{Cov}(\varepsilon)$ with the caps $C(\rho, \mathbf{x})$. Therefore, $\{\mathbf{x}\}$ also covers $S_{r}^{n}$ with unit caps.

Main proofs. To prove Theorem 1, we first observe (by numerical comparison) that the existing bound (16) is tighter for $n \leq 100$ than bound (5) of Theorem 1 or even its refined version (39) considered below. Thus, Theorem 1 holds for $n \leq 100$. For this reason, we shall only consider dimensions $n \geq 100$. In the end of the proof, we also address the asymptotic case $n \rightarrow \infty$, wherein the calculations are more straightforward. The proof is based on three lemmas.

Consider two caps $C(\mu, \mathbf{Z})$ and $C(\rho, \mathbf{Y})$ with centers $\mathbf{Y}$ and $\mathbf{Z}$, which are $d$-close. These caps are represented in Fig. 1. Here the origin $\mathbf{O}$ is the center of $S_{r}^{n}$.

Lemma 6 For any cap $C(\mu, \mathbf{Z})$, a randomly chosen $d$-close cap $C(\rho, \mathbf{Y})$ fails to cover any given point $\mathbf{x}$ of $C(\mu, \mathbf{Z})$ with probability $p(\mathbf{x}) \leq \omega$, where

$$
\omega=\frac{1}{4 \ln n}\left(1-\frac{3}{n} \ln ^{2} n\right)^{\frac{n-1}{2}} \leq \frac{1}{4 \ln n} \exp \left(-\frac{3}{2} \ln ^{2} n\right) .
$$

Proof The caps $C(\mu, \mathbf{Z})$ and $C(\rho, \mathbf{Y})$ have bases PQRSA and PMRTB, which form the balls $B_{\mu}^{n}(\mathbf{A})$ and $B_{\rho}^{n}(\mathbf{B})$. Below we consider the boundary PQRS of $C(\mu, \mathbf{Z})$, which forms the sphere $S_{\mu}^{n-1}(\mathbf{A})$. The bigger cap $C(\rho, \mathbf{Y})$ covers this boundary, with the exception of the cap PQR centered at $Q$. We first consider the case, when $\mathbf{x}$ is a boundary point and belongs to PQRS. Then the probability $p(\mathbf{x})$ is the fraction $\Omega$ of the entire boundary contained in uncovered cap PQR. We first estimate the half-angle $\alpha=\measuredangle \mathbf{P A Q}$ formed by the cap PQR. 
Let $d(\mathbf{H}, \mathbf{G})$ denote the distance between any two points $\mathbf{H}$ and $\mathbf{G}$. Also, let $\sigma(\mathbf{H})$ be the distance from a point $\mathbf{H}$ to the line $\mathbf{O B Y}$ that connects the origin $\mathbf{O}$ with the center $\mathbf{B}$ of the bigger base $B_{\rho}^{n}(\mathbf{B})$ and with the center $\mathbf{Y}$ of the cap $C(\rho, \mathbf{Y})$. We use inequalities

$$
\sigma(\mathbf{A}) \leq \sigma(\mathbf{Z}) \leq d(\mathbf{Z}, \mathbf{Y}) \leq d .
$$

On the other hand, consider the base PNR of the uncovered cap PQR. Here $\mathbf{N}$ denotes the center of this base. Then both lines $\mathbf{A N}$ and $\mathbf{B N}$ are orthogonal to this base. Also, $d(\mathbf{B}, \mathbf{P})=\rho$, and $d(\mathbf{N}, \mathbf{P}) \leq d(\mathbf{A}, \mathbf{P})=\mu$. Thus,

$$
d(\mathbf{B}, \mathbf{N})=\sqrt{d^{2}(\mathbf{B}, \mathbf{P})-d^{2}(\mathbf{N}, \mathbf{P})} \geq \sqrt{\rho^{2}-\mu^{2}} \geq \rho-\mu^{2} .
$$

The latter inequality follows from the trivial inequality $2 \rho-1 \geq \mu^{2}$. Finally, the center line OBY is orthogonal to the entire base PMRTB and its line BN. Then $d(\mathbf{B}, \mathbf{N})=\sigma(\mathbf{N})$ and

$$
d(\mathbf{A}, \mathbf{N}) \geq \sigma(\mathbf{N})-\sigma(\mathbf{A}) \geq \rho-\mu^{2}-d \geq \varepsilon .
$$

Now we consider the right triangle ANP and deduce that

$$
\cos \alpha=d(\mathbf{A}, \mathbf{N}) / d(\mathbf{A}, \mathbf{P}) \geq \varepsilon / \mu=\sqrt{\frac{3}{n}} \ln n .
$$

(Note that the latter expression exceeds 1 for $n<42$, which simply implies that a $d$-close cap entirely covers $\mu$-cap for small $n$.) Given $\alpha$, we can estimate the fraction $\Omega$ of the boundary sphere $S_{\mu}^{n-1}(\mathbf{A})$ contained in the uncovered cap PQR. This fraction can be defined as (see [4])

$$
\Omega<\{2 \pi(n-1)\}^{-1 / 2} \frac{\sin ^{n-1} \alpha}{\cos \alpha}<\left\{6 \pi \frac{n-1}{n}\right\}^{-1 / 2} \frac{\sin ^{n-1} \alpha}{\ln n}<\frac{\sin ^{n-1} \alpha}{4 \ln n} .
$$

The last inequality follows from the fact that $6 \pi\left(1-\frac{1}{n}\right)>16$. Now we use (30) and approximation $1-x \leq e^{-x-x^{2} / 2}$. This gives inequalities

$$
\begin{aligned}
\sin ^{n-1} \alpha & \leq\left(1-\frac{3}{n} \ln ^{2} n\right)^{\frac{n-1}{2}} \leq \exp \left\{\left(-\frac{3}{2} \ln ^{2} n-\frac{9}{4 n} \ln ^{4} n\right)\left(1-\frac{1}{n}\right)\right\} \\
& \leq \exp \left(-\frac{3}{2} \ln ^{2} n\right) \\
\Omega & \leq \frac{1}{4 \ln n} \exp \left(-\frac{3}{2} \ln ^{2} n\right) .
\end{aligned}
$$

Finally, consider the second case, when a point $\mathbf{x}$ does not belong to the boundary PQRS. Therefore, $\mathbf{x}$ is taken from a smaller cap $C\left(\mu^{\prime}, \mathbf{Z}\right) \subset C(\mu, \mathbf{Z})$ with the same center $\mathbf{Z}$ and a half-chord $\mu^{\prime}<\mu$. Similarly to the first case, we define the boundary $S_{\mu^{\prime}}^{n-1}\left(\mathbf{A}^{\prime}\right)$ of $C\left(\mu^{\prime}, \mathbf{Z}\right)$, where $\mathbf{A}^{\prime}$ is some center on the line $\mathbf{A Z}$. Again, $p(\mathbf{x})$ is the fraction $\Omega^{\prime}$ of this boundary left uncovered by the bigger cap $C(\rho, \mathbf{Y})$. To obtain the 
upper bound on $\Omega^{\prime}$, we only need to replace $\mu$ with a smaller $\mu^{\prime}$ in (30). This gives the smaller angle $\alpha^{\prime} \leq \arccos \left(\varepsilon / \mu^{\prime}\right)$, which is reduced to 0 if $\varepsilon \geq \mu^{\prime}$. In particular, the center $\mathbf{Z}$ of the cap is always covered by any $d$-close cap. Thus, we see that any internal layer $S_{\mu^{\prime}}^{n-1}\left(\mathbf{A}^{\prime}\right)$ of the cap $C(\mu, \mathbf{Z})$ has a smaller uncovered fraction $\Omega^{\prime} \leq \Omega$. This gives the required condition

$$
p(\mathbf{x}) \leq \Omega^{\prime} \leq \Omega<\omega
$$

for any point $\mathbf{x}$ and proves our lemma.

Remark Our choice of $d$ in (22) is central to the above proof, and even a marginal increase in $d$ will completely change our setting. Namely, it can be proven that about half the base of the $\mu$-cap is uncovered if a $\rho$-cap is $(d+\varepsilon)$-close.

Our next goal is to estimate the expected number $N^{\prime}$ of non-saturated caps $C\left(\mu, \mathbf{z}^{\prime}\right)$ left after $N$ trials.

Lemma 7 For $n \geq 100$, the expected number $N^{\prime}$ of non-saturated caps $C\left(\mu, \mathbf{z}^{\prime}\right)$ is

$$
N^{\prime}<2^{-n / 4} N
$$

Proof Given any center $\mathbf{z}$, a randomly chosen center $\mathbf{y}$ is $d$-close to $\mathbf{z}$ with the probability $\Omega_{d}$. Then the probability to obtain $s$ or fewer such caps is

$$
P=\sum_{i=0}^{s}\left(\begin{array}{c}
N \\
i
\end{array}\right) \Omega_{d}^{i}\left(1-\Omega_{d}\right)^{N-i} .
$$

Note that for any $i \leq s$,

$$
\left(1-\Omega_{d}\right)^{N-i} \leq \exp \left\{-\left(\Omega_{d}+\Omega_{d}^{2} / 2\right)(N-i)\right\} .
$$

Now we use inequality $\Omega_{d} \leq 1 / 2$ and the lower bound $N \Omega_{d}>\lambda n \ln n-\Omega_{d}$ of (25). Then

$$
\begin{aligned}
& \left(\Omega_{d}+\Omega_{d}^{2} / 2\right)(N-i) \\
& \quad=N \Omega_{d}\left(1+\frac{\Omega_{d}}{2}\right)-i \Omega_{d}\left(1+\frac{\Omega_{d}}{2}\right) \\
& \quad \geq\left(\lambda n \ln n-\Omega_{d}\right)+\frac{\Omega_{d}}{2}\left[\lambda n \ln n-\Omega_{d}-s\left(2+\Omega_{d}\right)\right] \geq \lambda n \ln n
\end{aligned}
$$

Here last inequality follows from the fact that $s\left(2+\Omega_{d}\right) \leq 5 n /(6 \ln \ln n)$ and therefore

$$
\lambda n \ln n-\Omega_{d}-s\left(2+\Omega_{d}\right) \geq 2 .
$$

Then

$$
P \leq e^{-\lambda n \ln n} \sum_{i=0}^{s} \frac{\left(\Omega_{d} N\right)^{i}}{i !} \leq e^{-\lambda n \ln n} \sum_{i=0}^{s} \frac{(\lambda n \ln n)^{i}}{i !}
$$


Note that consecutive summands in (32) differ at least $(\lambda n \ln n) / s \geq \lambda q \ln n$ times. Therefore

$$
\begin{aligned}
\sum_{i=0}^{s} \frac{(\lambda n \ln n)^{i}}{i !} & \leq \frac{(\lambda n \ln n)^{s}}{s !} \sum_{i=0}^{\infty}(\lambda q \ln n)^{-i} \\
& \leq 2 \frac{(\lambda n \ln n)^{s}}{s !} \leq \frac{(\lambda n \ln n)^{s}}{(s / e)^{s}} \leq(e \lambda q \ln n)^{n / q}
\end{aligned}
$$

Here the sum of the geometric series $(\lambda q \ln n)^{-i}$ was first bounded from above by $c_{n}<c_{100}<2$. Then we used the Sterling formula in the form $s !>(2 \pi s)^{1 / 2}(s / e)^{s}$ and removed (for simplicity) the vanishing term $2(2 \pi s)^{-1 / 2}$. Finally, the last inequality follows from the fact that its left-hand side increases in $s$ for any $s<\lambda n \ln n$. Summarizing, these substitutions give

$$
P \leq \exp \left\{n h_{n}-\lambda n \ln n\right\}
$$

where

$$
h_{n}=\frac{\ln (e \lambda q \ln n)}{q}=\frac{1}{3}+\frac{\ln (e \lambda q)}{q} .
$$

Next, we recalculate $\vartheta_{*}$ of (16) using parameter $\lambda$ of (22) and the lower bound of (25). It is easy to verify that for any $n$,

$$
\vartheta_{*} \leq 2(\lambda n \ln n-1 / 2) \leq 2 \Omega_{d} N
$$

Now we estimate the size of $\operatorname{Cov}(\mu)$ using (8) and (22) as follows

$$
|\operatorname{Cov}(\mu)| \leq \vartheta_{*} / \Omega_{\mu} \leq 2 N \Omega_{d} / \Omega_{\mu} \leq 2 N \mu^{n} \leq 2 N \exp \{n[\beta \ln n+\ln (12) / 2]\} .
$$

Thus, the expected number of non-saturated caps is

$$
\begin{aligned}
N^{\prime} & \leq|\operatorname{Cov}(\mu)| P \leq 2 N \exp \left\{n\left[h_{n}-(\lambda-\beta) \ln n+\ln (12) / 2\right]\right\} \\
& \leq 2 N \exp \left\{n\left[h_{n}-(5-\ln 12) / 2\right]\right\} .
\end{aligned}
$$

Now we see that the quantity $\Psi_{n}$ in the brackets of (34) consists of the declining positive function $h_{n}$ and the negative constant. Thus, $\Psi_{n}$ is a declining function of $n$. Direct calculation shows that $\Psi_{100}<-0.257$. Therefore estimate (31) holds.

Consider now the saturated caps $C(\mu, \mathbf{z})$ and the centers $\mathbf{u} \in \operatorname{Cov}(\varepsilon)$ inside them.

Lemma 8 For any $n \geq 100$, the number of centers $\mathbf{u}^{\prime \prime} \in \operatorname{Cov}(\varepsilon)$ left uncovered in all saturated caps $C(\mu, \mathbf{z})$ has expectation

$$
N^{\prime \prime}<2^{-n / 2} N
$$

Proof We first estimate the total number $|\operatorname{Cov}(\varepsilon)|$ of centers $\mathbf{u}$. Similarly to (33),

$$
|\operatorname{Cov}(\varepsilon)| \leq \vartheta_{*} / \Omega_{\varepsilon} \leq 2 N \Omega_{d} / \Omega_{\varepsilon} \leq 2 N(2 n \ln n)^{n} .
$$


Any cap $C(\mu, \mathbf{z})$ intersects with at least $s+1$ randomly chosen caps $C(\rho, \mathbf{y})$. According to Lemma 6 , any single $\rho$-cap fails to cover any given point $x \in C(\mu, \mathbf{z})$ with probability $\omega$ or less. Therefore any point $\mathbf{u}^{\prime \prime} \in C(\mu, \mathbf{z})$ is not covered with probability $\omega^{s+1}$ or less. Note that $\omega^{s+1}<\omega^{n / q}$ where $q=3 \ln \ln n$. Then we use the upper bound (27) for $\omega$ and deduce that

$$
N^{\prime \prime} \leq|\operatorname{Cov}(\varepsilon)| \cdot \omega^{n / q} \leq 2 N \exp \left\{-n \Phi_{n}\right\}
$$

where

$$
\Phi_{n}=\left(\frac{\ln ^{2} n}{2 \ln \ln n}+\frac{\ln 4}{3 \ln \ln n}\right)-\ln n-\ln \ln n-\ln 2+\frac{1}{3} .
$$

Direct calculation shows that $\Phi_{100}>0.71$. It is also easy to see that the first term of $\Phi_{n}$ (in parentheses) moderates both terms $\ln n$ and $\ln \ln n$. Thus, $\Phi_{n}>0.71$ for all $n \geq 100$, and the lemma is proved.

Proof of Theorem 1 Consider any cap $C(\mu, \overline{\mathbf{z}})$ that contains at least one uncovered center $\mathbf{u} \in \operatorname{Cov}(\varepsilon)$. Such a cap is either non-saturated or porous and therefore $\{\overline{\mathbf{z}}\}=\left\{\mathbf{z}^{\prime}\right\} \cup\left\{\mathbf{z}^{\prime \prime}\right\}$. (Equivalently, we can directly consider the set $\left\{\mathbf{z}^{\prime}\right\} \cup\left\{\mathbf{u}^{\prime \prime}\right\}$.) Then, according to Lemmas 7 and $8,\{\overline{\mathbf{z}}\}$ has expected size

$$
\bar{N} \leq N^{\prime}+N^{\prime \prime}<2^{1-n / 4} N .
$$

Thus, there exist $N$ randomly chosen centers $\mathbf{y}$ that leave at most $2^{1-n / 4} N$ centers $\overline{\mathbf{z}}$. The extended set $\{\mathbf{x}\}=\{\mathbf{y}\} \cup\{\overline{\mathbf{z}}\}$ forms a unit covering of $S_{r}^{n}$. This covering has density

$$
\vartheta \leq \Omega_{1}(N+\bar{N}) \leq \Omega_{1} N\left(1+2^{1-n / 4}\right) \leq \lambda n \ln n\left(1+2^{1-n / 4}\right) \Omega_{1} / \Omega_{d} .
$$

Similarly to inequality (9), we now verify that for $n \geq 100$,

$$
\Omega_{1} / \Omega_{d} \leq\left(1-\frac{1}{n \ln n}-n^{-2 \beta}\right)^{-n}<1+\frac{1}{\ln n}+\frac{1}{\ln ^{2} n}
$$

Indeed, replace parameter $t=\frac{1}{\ln n}$ used in the proof of Lemma 4 with the new value

$$
t=\frac{1}{\ln n}+n^{1-2 \beta}=\frac{1}{\ln n}+\frac{1}{\ln ^{4} n} .
$$

This new $t$ also satisfies inequality (11) for $n \geq 100$, which in turn proves inequality (37). As a secondary remark, note that (37) also proves asymptotic equality (24).

Finally, we take $\lambda$ of (9) and combine the last inequalities for $\vartheta$ and $\Omega_{1} / \Omega_{d}$ as follows

$$
\begin{aligned}
\frac{\vartheta}{n \ln n} & \leq\left(\frac{1}{2}+\frac{2 \ln \ln n}{\ln n}+\frac{5}{2 \ln n}\right)\left(1+\frac{1}{\ln n}+\frac{1}{\ln ^{2} n}\right)\left(1+2^{1-n / 4}\right) \\
& <\frac{1}{2}+\frac{2 \ln \ln n}{\ln n}+\frac{5}{\ln n} .
\end{aligned}
$$


Here we again used Lemma 3. Namely, we verify numerically that the last expression exceeds the previous one at $n=100$ and moderates it for larger $n$, due to its bigger remaining term $5 / \ln n$. This completes the non-asymptotic case $n \geq 3$.

To complete the proof of Theorem 1, we now present similar estimates for $n \rightarrow \infty$. We take any constant $b>3 / 2$ and redefine the parameters in (22) and (26) as follows:

$$
\begin{aligned}
& \beta=\frac{1}{2}+b \frac{\ln \ln n}{\ln n}, \\
& \lambda=\beta+\frac{3}{4} \frac{1}{\ln n}, \\
& \mu=\frac{1}{2 \sqrt{n}} \ln ^{-b} n, \\
& q=\ln ^{2} \ln n .
\end{aligned}
$$

First, bounds (30) and (27) can be replaced with

$$
\begin{gathered}
\cos \alpha \geq \varepsilon / \mu \geq \frac{1}{\sqrt{n}} \ln ^{b-1} n, \\
\omega \leq\left(1-\frac{1}{n} \ln ^{2 b-2} n\right)^{\frac{n-1}{2}} \leq \exp \left(-\frac{1}{2} \ln ^{2 b-2} n\right) .
\end{gathered}
$$

Second, bound (34) can be rewritten as

$$
N^{\prime} \leq 2 N \exp \left\{n\left(\frac{\ln (e \lambda q \ln n)}{q}+\ln 2-\frac{3}{4}\right)\right\}
$$

Note that the first term $\ln (e \lambda q \ln n) / q$ vanishes for $n \rightarrow \infty$, and $N^{\prime}$ declines exponentially in $n$. Thus, Lemma 7 holds. Finally, bound (35) is replaced with

$$
N^{\prime \prime} \leq 2 N \exp \left\{n\left[\ln (2 n \ln n)-\frac{1}{2} \frac{\ln ^{2 b-2} n}{\ln ^{2} \ln n}\right]\right\}
$$

which vanishes (faster than exponent in $n$ ) for any given $b>3 / 2$. Thus, Lemma 8 also holds. Then we proceed similarly to (37). In this case, for sufficiently large $n$, we obtain the density

$$
\frac{\vartheta}{n \ln n} \leq\left(\frac{1}{2}+b \frac{\ln \ln n}{\ln n}+\frac{3}{4} \frac{1}{\ln n}\right)\left(1+\frac{1}{\ln n}+\frac{1}{\ln ^{2} n}\right) \leq \frac{1}{2}+b \frac{\ln \ln n}{\ln n}+\frac{3}{2} \frac{1}{\ln n},
$$

which proves (6). This completes the proof of Theorem 1.

For finite $n$, we can slightly refine bounds (28-29), and also use exact expression for $\omega$ in (27). However, these refinements only marginally improve the main bound (5), which is replaced with

$$
\frac{\vartheta}{n \ln n} \leq \frac{1}{2}+\frac{2 \ln \ln n}{\ln n}+\frac{4 \ln \ln n}{\ln n} .
$$


Finally, note that Theorem 1 directly leads to Corollary 2. Indeed, here we can use the well known fact

$$
\vartheta\left(\mathbb{R}^{n-1}\right)=\lim _{r \rightarrow \infty} \vartheta\left(S_{r}^{n}\right)
$$

(see a sketch of the proof in [6] or Theorem $\Pi .1$ in [7], where a similar proof is detailed for packings of $\mathbb{R}^{n}$ ).

Concluding remarks In summary, we prove in this paper that the classic Rogers bound (3) on covering density of a sphere $S_{r}^{n}$ or the Euclidean space $\mathbb{R}^{n}$ can be reduced about two times for large dimensions $n$. The main open problem is to further reduce the gap between this bound and its lower counterpart (2), which is linear in $n$. In this regard, note that our design holds if we employ any constant parameter $\beta>1 / 2$ introduced in (22). However, it can be verified that choosing a smaller constant $\beta<1 / 2$ will first increase parameter $\mu=n^{-\beta}$ and then reduce parameter $d$ of (22) to the order of $1-\mu^{2} / 2$. The latter reduction will exponentially increase the covering size, by a factor of

$$
\Omega_{\rho} / \Omega_{d} \rightarrow \exp \{1-2 \beta\}
$$

Therefore, our conjecture is that a completely new design is needed for further asymptotic reductions.

Another important problem is to extend the above results to the balls $B_{r}^{n}$ of an arbitrary radius $r$. Our conjecture is that $\vartheta\left(B_{r}^{n}\right) \leq\left(\frac{1}{2}+o(1)\right) n \ln n$ for any $r$ and $n \rightarrow \infty$.

Acknowledgements The author is grateful to an anonymous referee for many helpful comments and remarks. This research was supported in part by NSF grants CCF-0622242 and CCF-0635339.

\section{References}

1. Coxeter, H.S.M., Few, L., Rogers, C.A.: Covering space with equal spheres. Mathematika 6, 147-157 (1959)

2. Rogers, C.A.: A note on coverings. Mathematika 4, 1-6 (1957)

3. Rogers, C.A.: Covering a sphere with spheres. Mathematika 10, 157-164 (1963)

4. Böröczky, K. Jr., Wintsche, G.: Covering the sphere with equal spherical balls. In: Aronov, B., Bazú, S., Sharir, M., Pach, J. (eds.) Discrete Computational Geometry-The Goldman-Pollack Festschrift, pp. 237-253. Springer, Berlin (2003)

5. Verger-Gaugry, J.-L.: Covering a ball with smaller equal balls in $\mathbb{R}^{n}$. Discrete Comput. Geom. 33, 143-155 (2005)

6. Conway, J.H., Sloane, N.J.A.: Sphere Packings, Lattices and Groups. Springer, New York (1988)

7. Levenstein, V.I.: Bounds on packings of metric spaces and some of their applications. Vopr. Kibern. 40, 43-109 (1983) (in Russian)

8. Dumer, I.: Covering spheres and balls with smaller balls. In: 2006 IEEE International Symposium Information Theory, Seattle, WA, July 9-15 2006, pp. 992-995 (2006) 